\title{
INVARIANTS OF SYSTEMS \\ OF LINEAR DIFFERENTIAL EQUATIONS
}

BY

\section{E. J. WILCZYNSKI}

The theory of the invariants of a single linear homogeneous differential equation rests upon StaEcKel's theorem, that the most general point-transformation which converts a general homogeneous linear differential equation of the $m$-th order $(m>1)$

$$
\frac{d^{m} y}{d x^{m}}+P_{1}(x) \frac{d^{m-1} y}{d x^{m-1}}+\cdots+P_{m}(x) y=0,
$$

into another equation of the same form and order, is

$$
x=f(\xi), \quad y=\eta g(\xi),
$$

where $f(\xi)$ and $g(\xi)$ are arbitrary functions of $\xi .^{*}$

Those functions of $P_{1}, P_{2}, \ldots, P_{m}$ and the derivatives of these quantities, which are the same for the equation (1) and for any equation obtained from (1) by a transformation (2), are called invariants of (1). Functions having this invariant property and containing also $y, d y / d x$, etc., are called covariants. The investigation of such invariants and covariants has led to many new and interesting results concerning the equation (1). This theory is associated with the names of Cockle, Malet, Laguerre, Halphen, Brioschi, Forsyth, and others.

The author has recently shown that the most general point-transformation, which converts a system of $n$ homogeneous linear differential equations into another of the same form and order, is

$$
x=f(\xi), \quad y_{k}=\sum_{i=1}^{n} a_{k i}(\xi) \eta_{i} \quad(k=1,2, \cdots, n),
$$

where $f(\xi)$ and $a_{k i}(\xi)$ are arbitrary functions of $\xi$, and the determinant $\left|a_{k i}(\xi)\right|$ does not ranish identically. $\dagger$

We shall consider, in this paper, those combinations of the coefficients of a system of linear differential equations which remain invariant when the system

* Crelle's Journal, vol. 111.

† American Journal of Mathematics, January, 1901. 
is transformed by the above transformation (3). These transformations obviously form an infinite continuous group, and we shall employ LiE's theory throughout, as has been done in the case of a single differential equation by Dr. Bouton.* We shall not, in this first paper, pay much attention to the applications of the theory, nor give more than a passing mention to covariants.

\section{§1.. Finite transformations of the dependent variables.}

We shall at first confine ourselves to the transformation of the dependent variables. Those functions of the coefficients of the system which remain invariant for all such transformations, may be called seminvariants. These are of considerable importance in themselves, and besides furnish the basis for the theory of invariants under the general transformation.

Let the given system be

$$
y_{i}^{(m)}+\sum_{l=0}^{m-1} \sum_{k=1}^{n} p_{i k l} y_{k}^{(l)}=0
$$$$
(i=1,2, \cdots, n)
$$

and let this system be transformed by the equations

$$
y_{k}=\sum_{\lambda=1}^{n} a_{k \lambda}(x) \eta_{\lambda}
$$$$
(k=1,2, \cdots, n),
$$

where $a_{k \lambda}(x)$ are arbitrary functions of $x$, and where the determinant

$$
\left|a_{k \lambda}(x)\right| \quad(k, \lambda=1,2, \cdots, n),
$$

does not vanish. As usual we denote derivatives by accents. Then we have from (2)

$$
y_{k}^{(l)}=\sum_{\lambda=1}^{n} \sum_{\rho=0}^{l}\left(\begin{array}{l}
l \\
\rho
\end{array}\right) a_{k \lambda}^{(\rho)} \eta_{\lambda}^{(l-\rho)} \quad(k=1,2, \ldots, n ; l=0,1,2, \ldots, m),
$$

where in general $\left(\begin{array}{l}l \\ \rho\end{array}\right)$ denotes the coefficient of $x^{\rho}$ in the expansion of $(1+x)^{l}$.

Equations (1) then become

(4) $\begin{array}{r}\sum_{\lambda=1}^{n} a_{i \lambda} \eta_{\lambda}^{(m)}+\sum_{\lambda=1}^{n} \sum_{\rho=1}^{m}\left(\begin{array}{c}m \\ \rho\end{array}\right) \alpha_{i \lambda}^{(\rho)} \eta_{\lambda}^{(m-\rho)}+\sum_{l=0}^{m-1} \sum_{k=1}^{n} \sum_{\mu=1}^{n} \sum_{\sigma=0}^{l}\left(\begin{array}{l}l \\ \sigma\end{array}\right) p_{i k l} a_{k \mu}^{(\sigma)} \eta_{\mu}^{(l-\sigma)}=0 \\ (i=1,2, \cdots, n) .\end{array}$

The coefficient of $\eta_{\mu}^{(\nu)}$ in the double sum is

$$
\left(\begin{array}{c}
m \\
m-\nu
\end{array}\right) \boldsymbol{a}_{i \mu}^{(m-\nu)}
$$

and in the quadruple sum, the coefficient of $\eta_{\mu}^{(\nu)}$ is

* American Journal of Mathematics, vol. 21, no. 2, 1899. 


$$
\begin{aligned}
\sum_{k=1}^{n}\left[\left(\begin{array}{l}
\nu \\
0
\end{array}\right) p_{i k \nu} a_{k \mu}+\left(\begin{array}{c}
\nu+1 \\
1
\end{array}\right) p_{i, k, \nu+1} a_{k \mu}^{(1)}\right. & +\cdots \\
& \left.+\left(\begin{array}{c}
m-1 \\
m-1-\nu
\end{array}\right) p_{i, k, m-1} a_{k \mu}^{(m-1-\nu)}\right]
\end{aligned}
$$

i. e.,

$$
\sum_{k=1}^{n} \sum_{\tau=0}^{m-1-\nu}\left(\begin{array}{c}
\nu+\tau \\
\tau
\end{array}\right) p_{i, k, \nu+\tau} a_{k \mu}^{(\tau)}
$$

Thus, from (4) we obtain

(5) $\sum_{\lambda=1}^{n} a_{i \lambda} \eta_{\lambda}^{(m)}+\sum_{\mu=1}^{n} \sum_{\nu=0}^{m-1} \eta_{\mu}^{(\nu)}\left[\left(\begin{array}{c}m \\ m-\nu\end{array}\right) a_{i \mu}^{(m-\nu)}\right.$

or if we put

$$
\left.+\sum_{k=1}^{n} \sum_{\tau=0}^{m-1-\nu}\left(\begin{array}{c}
\nu+\tau \\
\tau
\end{array}\right) p_{i, k, \nu+\tau} a_{k \mu}^{(\tau)}\right]=0 \quad(i=1,2, \cdots, n),
$$

$$
\Delta=\left|a_{i \lambda}\right|
$$

$(i, \lambda=1,2, \cdots, n)$

and denote by $A_{i \lambda}$ the minor of $a_{i \lambda}$ in this determinant,

$$
\begin{aligned}
\Delta \eta_{\lambda}^{(m)}+\sum_{\mu=1}^{n} \sum_{\nu=0}^{m-1} \eta_{\mu}^{(\nu)} \sum_{i=1}^{n} A_{i \lambda}\left[\left(\begin{array}{c}
m \\
m-\nu
\end{array}\right) a_{i \mu}^{(m-\nu)}\right. \\
\left.\quad+\sum_{k=1}^{n} \sum_{\tau=0}^{m-1-\nu}\left(\begin{array}{c}
\nu+\tau \\
\tau
\end{array}\right) p_{i, k, \nu+\tau} a_{k \mu}^{(\tau)}\right]=0 \quad(\lambda=1,2, \cdots, n) .
\end{aligned}
$$

If then we write this system in the form

we have

$$
\eta_{\lambda}^{(m)}+\sum_{\nu=0}^{m-1} \sum_{\mu=1}^{n} \pi_{\lambda \mu \nu} \eta_{\mu}^{(\nu)}=0 \quad(\lambda=1,2, \cdots, n),
$$

$$
\begin{array}{r}
\Delta \pi_{\lambda \mu \nu}=\sum_{i=1}^{n} A_{i \lambda}\left[\left(\begin{array}{c}
m \\
m-\nu
\end{array}\right) a_{i \mu}^{(m-\nu)}+\sum_{k=1}^{n} \sum_{\tau=0}^{m-1-\nu}\left(\begin{array}{c}
\nu+\tau \\
\tau
\end{array}\right) p_{i, k, \nu+\tau} a_{k \mu}^{(\tau)}\right] \\
(\lambda, \mu=1,2, \cdots, n ; \nu=0,1, \ldots, m-1) .
\end{array}
$$

Thus, if (1) is transformed into (8) by transformations (2), the relations between the coefficients of (1) and (8) are the equations (9).

Equations (9) represent an infinite continuous group, isomorphic with the group represented by equations (2). For to every transformation of the latter group corresponds one of the former, and they obviously have the group property. Both groups can be defined by differential equations, so that LIE's theory of infinite groups may be applied.

\section{§2. Infinitesimal transformations of the dependent variables.}

We proceed to consider the infinitesimal transformations of our infinite group. The variables $y_{1}, y_{2}, \ldots, y_{n}$ will undergo the most general infinitesinal transformation of form (2) if we put 


$$
\begin{aligned}
a_{i i}(x)=1+\phi_{i i}(x) \delta t, \quad a_{i k}(x)= & \phi_{i k}(x) \delta t \\
& (i \neq k),(i, k=1,2, \cdots, n),
\end{aligned}
$$

where $\delta t$ is an infinitesimal and the $\phi_{i k}$ 's are arbitrary functions of $x$. We wish to find the corresponding infinitesimal transformations of the coefficients $p_{i k l}$.

Neglecting infinitesimals of order higher than the first, we find

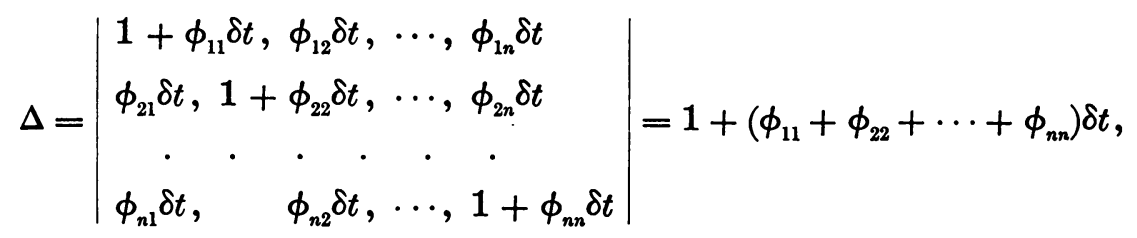

and similarly

$$
\left\{\begin{array}{l}
A_{i i}=1+\left(\phi_{11}+\phi_{22}+\cdots+\phi_{n n}-\phi_{i i}\right) \delta t, \\
A_{k i}=-\phi_{i k} \delta t
\end{array}\right.
$$

Substituting these values in (9), we have

$$
\begin{aligned}
& \Delta \pi_{\lambda \mu \nu}=\sum_{i=1}^{n}-\phi_{\lambda i} \delta t\left[\left(\begin{array}{c}
m \\
m-\nu
\end{array}\right) \phi_{i \mu}^{(m-\nu)} \delta t+\sum_{k=1}^{n} \sum_{\tau=0}^{m-1-\nu}\left(\begin{array}{c}
\nu+\tau \\
\tau
\end{array}\right) p_{i, k, \nu+\tau} \phi_{k \mu}^{(\tau)} \delta t\right. \\
& \left.+p_{i \mu \nu}\right]+\left[1+\left(\phi_{11}+\phi_{22}+\cdots+\phi_{n n}\right) \delta t\right]\left[\left(\begin{array}{c}
m \\
m-\nu
\end{array}\right) \phi_{\lambda \mu}^{(m-\nu)} \delta t\right. \\
& \left.+\sum_{k=1}^{n} \sum_{\tau=0}^{m-1-\nu}\left(\begin{array}{c}
\nu+\tau \\
\tau
\end{array}\right) p_{\lambda, k, \nu+\tau} \phi_{k \mu}^{(\tau)} \delta t+p_{\lambda \mu \nu}\right]
\end{aligned}
$$

or

$$
\begin{aligned}
\Delta \pi_{\lambda \mu \nu} & =p_{\lambda \mu \nu}-\sum_{i=1}^{n} \phi_{\lambda i} p_{i \mu \nu} \delta t+\left(\begin{array}{c}
m \\
m-\nu
\end{array}\right) \phi_{\lambda \mu}^{(m-\nu)} \delta t \\
& +\sum_{k=1}^{n} \sum_{\tau=0}^{m-1-\nu}\left(\begin{array}{c}
\nu+\tau \\
\tau
\end{array}\right) p_{\lambda, k, \nu+\tau} \phi_{k \mu}^{(\tau)} \delta t+p_{\lambda \mu \nu}\left(\phi_{11}+\phi_{22}+\cdots+\phi_{n n}\right) \delta t .
\end{aligned}
$$

Dividing by $\Delta=1+\left(\phi_{11}+\cdots+\phi_{n n}\right) \dot{\delta t}$, and denoting the infinitesimal difference $\pi_{\lambda \mu \nu}-p_{\lambda \mu \nu}$ by $\delta p_{\lambda \mu \nu}$, we find

$$
\begin{aligned}
& \frac{\delta p_{\lambda \mu \nu}}{\delta t}=\sum_{k=1}^{n}\left(\phi_{k \mu} p_{\lambda k \nu}-\phi_{\lambda k} p_{k \mu \nu}\right) \\
& +\sum_{k=1}^{n} \sum_{\tau=1}^{m-1-\nu}\left(\begin{array}{c}
\nu+\tau \\
\tau
\end{array}\right) \phi_{k \mu}^{(\tau)} p_{\lambda, k, \nu+\tau}+\left(\begin{array}{c}
m \\
m-\nu
\end{array}\right) \phi_{\lambda \mu}^{(m-\nu)}, \\
& (\lambda, \mu=1,2, \cdots, n ; \nu=0,1,2, \cdots, m-1) .
\end{aligned}
$$


These are the required infinitesimal transformations of $p_{\lambda \mu \nu}$. Those of $p_{\lambda \mu \nu}^{\prime}$, $p_{\lambda \mu \nu}^{\prime \prime}$, etc., may be obtained from (14) by differentiation.

\section{§3. Calculation of the seminvariants for $m=n=2$.}

The complexity of the general problem is so great that it appears wise to limit the further discussion in this paper to the very special case $m=n=2$. This will throw considerable light on the general case.

Let us put, in this case, for abbreviation

$$
\left\{\begin{array}{l}
p_{\lambda, \mu, m-1}=p_{\lambda \mu 1}=p_{\lambda \mu}, \quad p_{\lambda, \mu, m-2}=p_{\lambda \mu 0}=q_{\lambda \mu}, \\
\frac{\partial f}{\partial p_{\lambda \mu}}=P_{\lambda \mu}, \quad \frac{\partial f}{\partial p_{\lambda \mu}^{\prime}}=P_{\lambda \mu}^{\prime}, \quad \frac{\partial f}{\partial q_{\lambda \mu}}=Q_{\lambda \mu}, \quad \text { etc. }
\end{array}\right.
$$

We have then, from (14),

$$
\left\{\begin{array}{l}
\frac{\delta p_{\lambda \mu}}{\delta t}=\sum_{k=1}^{2}\left(\phi_{k \mu} p_{\lambda k}-\phi_{\lambda k} p_{k \mu}\right)+2 \phi_{\lambda \mu}^{\prime} \\
\frac{\delta p_{\lambda \mu}^{\prime}}{\delta t}=\sum_{k=1}^{2}\left(\phi_{k \mu} p_{\lambda k}^{\prime}-\phi_{\lambda k} p_{k \mu}^{\prime}+\phi_{k \mu}^{\prime} p_{\lambda k}-\phi_{\lambda k}^{\prime} p_{k \mu}\right)+2 \phi_{\lambda \mu}^{(2)} \\
\frac{\delta q_{\lambda \mu}}{\delta t}=\sum_{k=1}^{2}\left(\phi_{k \mu} q_{\lambda k}-\phi_{\lambda k} q_{k \mu}\right)+\sum_{k=1}^{2} \phi_{k \mu}^{\prime} p_{\lambda k}+\phi_{\lambda \mu}^{(2)}
\end{array}\right.
$$

Now if $f$ is a seminvariant depending only upon $p_{\lambda \mu}, p_{\lambda \mu}^{\prime}, q_{\lambda \mu}$, we must have

$$
\sum_{\lambda, \mu}\left(P_{\lambda \mu} \delta p_{\lambda \mu}+P_{\lambda \mu}^{\prime} \delta p_{\lambda \mu}^{\prime}+Q_{\lambda \mu} \delta q_{\lambda \mu}\right)=0
$$

for all values of $\phi_{r s}, \phi_{r s}^{\prime}, \phi_{r s}^{\prime \prime}$. Putting the coefficients of these twelve arbitrary functions equal to zero, we obtain the following system of partial differential equations for such seminvariants :

$$
\begin{cases}2 P_{r s}^{\prime}+Q_{r s}=0, & \\ 2 P_{r s}+\sum_{\lambda=1}^{2}\left(p_{\lambda r} P_{\lambda s}^{\prime}-p_{s \lambda} P_{r \lambda}^{\prime}+p_{\lambda r} Q_{\lambda \dot{s}}\right)=0, & (r, s=1,2) . \\ \sum_{\lambda=1}^{2}\left(p_{\lambda r} P_{\lambda s}-p_{s \lambda} P_{r \lambda}+p_{\lambda r}^{\prime} P_{\lambda s}^{\prime}-p_{s \lambda}^{\prime} P_{r \lambda}^{\prime}+q_{\lambda r} Q_{\lambda s}-q_{s \lambda} Q_{r \lambda}\right)=0 .\end{cases}
$$

This is a complete system of twelve equations with twelve independent variables. But there are two relations among them, so that we shall have two independent solutions, i. e., two independent seminvariants containing only the variables $p_{\lambda \mu}, p_{\lambda \mu}^{\prime}, q_{\lambda \mu}$.

The first four equations of the system tell us that the quantities $p_{r \text {, }}^{\prime}$ and $q_{r \text { r }}$ can occur only in combinations

$$
2 p_{r s}^{\prime}-4 q_{r s}
$$


The next four equations, written separately, are

$$
\left\{\begin{array}{l}
2 P_{12}+\left(p_{11}-p_{22}\right) P_{12}^{\prime}+p_{21}\left(P_{22}^{\prime}-P_{11}^{\prime}\right)+p_{11} Q_{12}+p_{21} Q_{22}=0 \\
2 P_{11}+p_{21} P_{21}^{\prime}-p_{12} P_{12}^{\prime}+p_{11} Q_{11}+p_{21} Q_{21}=0 \\
2 P_{22}+p_{12} P_{12}^{\prime}-p_{21} P_{21}^{\prime}+p_{12} Q_{12}+p_{22} Q_{22}=0 \\
2 P_{21}+\left(p_{22}-p_{11}\right) P_{21}^{\prime}+p_{12}\left(P_{11}^{\prime}-P_{22}^{\prime}\right)+p_{12} Q_{11}+p_{22} Q_{21}=0
\end{array}\right.
$$

They show that the only possible combinations of $p_{r s}, p_{r s}^{\prime}, q_{r s}$ are

$$
\left\{\begin{array}{l}
u_{11}=2 p_{11}^{\prime}-4 q_{11}+p_{11}^{2}+p_{12} p_{21} \\
u_{12}=2 p_{12}^{\prime}-4 q_{12}+p_{12}\left(p_{11}+p_{22}\right) \\
u_{21}=2 p_{21}^{\prime}-4 q_{21}+p_{21}\left(p_{11}+p_{22}\right) \\
u_{22}=2 p_{22}^{\prime}-4 q_{22}+p_{22}^{2}+p_{12} p_{21}
\end{array}\right.
$$

so that the seminvariants, here considered, are functions of $u_{11}, u_{12}, u_{21}, u_{22}$ only.

The last four equations (18) are

$$
\left\{\begin{array}{c}
U_{1} \equiv\left(p_{11}-p_{22}\right) P_{12}+p_{21}\left(P_{22}-P_{11}\right)+\left(p_{11}^{\prime}-p_{22}^{\prime}\right) P_{12}^{\prime} \\
\quad+p_{21}^{\prime}\left(P_{22}^{\prime}-P_{11}^{\prime}\right)+\left(q_{11}-q_{22}\right) Q_{12}+q_{21}\left(Q_{22}-Q_{11}\right)=0 \\
U_{2} \equiv p_{21} P_{21}-p_{12} P_{12}+p_{21}^{\prime} P_{21}^{\prime}-p_{12}^{\prime} P_{12}^{\prime}+q_{21} Q_{21}-q_{12} Q_{12}=0, \\
U_{3} \equiv p_{12} P_{12}-p_{21} P_{21}+p_{12}^{\prime} P_{12}^{\prime}-p_{21}^{\prime} P_{21}^{\prime}+q_{12} Q_{12}-q_{21} Q_{21}=0 \\
U_{4} \equiv\left(p_{22}-p_{11}\right) P_{21}+p_{12}\left(P_{11}-P_{22}\right)+\left(p_{22}^{\prime}-p_{11}^{\prime}\right) P_{21}^{\prime} \\
\quad+p_{12}^{\prime}\left(P_{11}^{\prime}-P_{22}^{\prime}\right)+\left(q_{22}-q_{11}\right) Q_{21}+q_{12}\left(Q_{11}-Q_{22}\right)=0,
\end{array}\right.
$$

with the obvious relation

$$
U_{2}+U_{3}=0 \text {. }
$$

But there is another relation between these four equations and the other eight, these latter being themselves obviously independent. If we compute from them $P_{r s}$ and $P_{r s}^{\prime}$ and substitute the values of these quantities, thus obtained, in (21), that system becomes

$$
\left\{\begin{array}{l}
\left(u_{11}-u_{22}\right) Q_{12}-u_{21}\left(Q_{11}-Q_{22}\right)=0, \\
-\left(u_{11}-u_{22}\right) Q_{21}+u_{12}\left(Q_{11}-Q_{22}\right)=0, \\
u_{12} Q_{12}-u_{21} Q_{21}=0,
\end{array}\right.
$$

where the last equation is a consequence of the other two.

The two independent solutions of this system are 


$$
I=u_{11}+u_{22}, \quad J=u_{11} u_{22}-u_{12} u_{21},
$$

and these are the seminvariants required.

Let us proceed to obtain next those seminvariants which contain also the quantities $p_{i k}^{\prime \prime}$ and $q_{i k}^{\prime}$. They must satisfy the following system of partial differential equations :

$$
\left\{\begin{array}{c}
\left(\begin{array}{l}
\text { (a) } 2 P_{r s}^{\prime \prime}+Q_{r s}^{\prime}=0, \\
\text { (b) } 2 P_{r s}^{\prime}+Q_{r s}+\sum_{\lambda=1}^{2}\left(p_{\lambda r} P_{\lambda s}^{\prime \prime}-p_{s \lambda} P_{r \lambda}^{\prime \prime}+p_{\lambda r} Q_{\lambda s}^{\prime}\right)=0, \\
(c) 2 P_{r s}+\sum_{\lambda=1}^{2}\left(p_{\lambda r} P_{\lambda s}^{\prime}-p_{s \lambda} P_{r \lambda}^{\prime}-2 p_{s \lambda}^{\prime} P_{r \lambda}^{\prime \prime}\right. \\
\left.\quad+q_{\lambda r} Q_{\lambda s}^{\prime}-q_{s \lambda} Q_{r \lambda}^{\prime}+p_{\lambda r} Q_{\lambda s}\right)=0 \\
\text { (d) } \sum_{\lambda=1}^{2}\left(p_{\lambda r} P_{\lambda s}-p_{s \lambda} P_{r \lambda}+p_{\lambda r}^{\prime} P_{\lambda s}^{\prime}-p_{s \lambda}^{\prime} P_{r \lambda}^{\prime}+p_{\lambda r}^{\prime \prime} P_{\lambda s}^{\prime \prime}\right. \\
\left.-p_{s \lambda}^{\prime \prime} P_{r \lambda}^{\prime \prime}+q_{\lambda r} Q_{\lambda s}-q_{s \lambda} Q_{r \lambda}+q_{\lambda r}^{\prime} Q_{\lambda s}^{\prime}-q_{s \lambda}^{\prime} Q_{r \lambda}^{\prime}\right)=0
\end{array}\right.
\end{array}\right.
$$

among which there is one relation. Thus there are 15 independent equations and 20 variables. There are therefore five seminvariants satisfying these equations. Of these we already know four, namely, $I, J, d I / d x, d J_{i} d x$, which are obviously independent.

Let us put

$$
u_{i k}^{\prime}=\frac{d u_{i k}}{d x}, \text { etc: }
$$

Then, since according to $(20)$, we have

therefore

$$
u_{i k}=2 p_{i k}^{\prime}-4 q_{i k}+\sum_{j=1}^{2} p_{i j} p_{j k},
$$

$$
u_{i k}^{\prime}=2 p_{i k}^{\prime \prime}-4 q_{i k}^{\prime}+\sum_{j=1}^{2}\left(p_{i j} p_{j k}^{\prime}+p_{i j}^{\prime} p_{j k}\right) \text {. }
$$

It will be easily proved from equations (25) (a) and (b) that our seminvariants are functions of the twelve arguments

$$
p_{i k}, \quad u_{i k}, \quad u_{i k}^{\prime} \text {. }
$$

Denoting the left members of (25) (c) by $\Omega_{1} \ldots \Omega_{4}$, so that

$$
\Omega_{1}=2 P_{11}+\cdots, \quad \Omega_{2}=2 P_{12}+\cdots, \quad \Omega_{3}=2 P_{21}+\cdots, \quad \Omega_{4}=2 P_{22}+\cdots,
$$

we find

$$
\begin{gathered}
\Omega_{1}\left(u_{i k}\right)=\Omega_{2}\left(u_{i k}\right)=\Omega_{3}\left(u_{i k}\right)=\Omega_{4}\left(u_{i k}\right)=0, \\
\left\{\begin{array}{llll}
\Omega_{1}\left(p_{11}\right)=2, & \Omega_{2}\left(p_{11}\right)=0, & \Omega_{3}\left(p_{11}\right)=0, & \Omega_{4}\left(p_{11}\right)=0, \\
\Omega_{1}\left(p_{12}\right)=0, & \Omega_{2}\left(p_{12}\right)=2, & \Omega_{3}\left(p_{12}\right)=0, & \Omega_{4}\left(p_{12}\right)=0, \\
\Omega_{1}\left(p_{21}\right)=0, & \Omega_{2}\left(p_{21}\right)=0, & \Omega_{3}\left(p_{21}\right)=2, & \Omega_{4}\left(p_{21}\right)=0, \\
\Omega_{1}\left(p_{22}\right)=0, & \Omega_{2}\left(p_{22}\right)=0, & \Omega_{3}\left(p_{22}\right)=0, & \Omega_{4}\left(p_{22}\right)=2,
\end{array}\right.
\end{gathered}
$$


and

$$
\left\{\begin{array}{lll}
\Omega_{1}\left(u_{11}^{\prime}\right)=0, & \Omega_{2}\left(u_{11}^{\prime}\right)=-u_{21}, & \\
\Omega_{3}\left(u_{11}^{\prime}\right)=+u_{12}, & \Omega_{4}\left(u_{11}^{\prime}\right)=0, \\
\Omega_{1}\left(u_{12}^{\prime}\right)=-u_{12}, & \Omega_{2}\left(u_{12}^{\prime}\right)=u_{11}-u_{22}, & \\
\Omega_{3}\left(u_{12}^{\prime}\right)=0, & \Omega_{4}\left(u_{12}^{\prime}\right)=+u_{12}, \\
\Omega_{1}\left(u_{21}^{\prime}\right)=+u_{21}, & \Omega_{2}\left(u_{21}^{\prime}\right)=0, & \\
& \Omega_{3}\left(u_{21}^{\prime}\right)=-\left(u_{11}-u_{22}\right), & \Omega_{4}\left(u_{21}^{\prime}\right)=-u_{21}, \\
\Omega_{1}\left(u_{22}^{\prime}\right)=0, & \Omega_{2}\left(u_{22}^{\prime}\right)=+u_{21}, & \\
\Omega_{3}\left(u_{22}^{\prime}\right)=-u_{12}, & \Omega_{4}\left(u_{22}^{\prime}\right)=0 .
\end{array}\right.
$$

From these equations it is easily seen that the eight independent functions of the arguments (28) which verify the equations $\Omega_{\lambda}=0$, are the quantities $u_{i k}$ and

$$
\left\{\begin{array}{l}
v_{11}=2 u_{11}^{\prime}+p_{12} u_{21}-p_{21} u_{12}, \\
v_{12}=2 u_{12}^{\prime}+\left(p_{11}-p_{22}\right) u_{12}-p_{12}\left(u_{11}-u_{22}\right), \\
v_{21}=2 u_{21}^{\prime}-\left(p_{11}-p_{22}\right) u_{21}+p_{21}\left(u_{11}-u_{22}\right), \\
v_{22}=2 u_{22}^{\prime}-p_{12} u_{21}+p_{21} u_{12} .
\end{array}\right.
$$

Denoting by $X_{1}, \cdots, X_{4}$ the left members of $(25)(d)$ we find :

$$
\left\{\begin{array}{ccc}
X_{1}\left(v_{11}\right)=0, & X_{2}\left(v_{11}\right)=-v_{21}, & \\
X_{3}\left(v_{11}\right)=+v_{12}, & X_{4}\left(v_{11}\right)=0, \\
X_{1}\left(v_{12}\right)=-v_{12}, & X_{2}\left(v_{12}\right)=v_{11}-v_{22}, & \\
X_{3}\left(v_{12}\right)=0, & X_{4}\left(v_{12}\right)=+v_{12}, \\
X_{1}\left(v_{21}\right)=+v_{21}, & X_{2}\left(v_{21}\right)=0, & \\
X_{3}\left(v_{21}\right)=-\left(v_{11}-v_{22}\right), & X_{4}\left(v_{21}\right)=-v_{21}, \\
X_{1}\left(v_{22}\right)=0, & X_{2}\left(v_{22}\right)=+v_{21}, & \\
& X_{3}\left(v_{22}\right)=-v_{12}, & X_{4}\left(v_{22}\right)=0,
\end{array}\right.
$$

the equations for $X_{1}\left(u_{i k}\right)$, etc., being precisely of the same form.

From (33) we find that

$$
v_{11}+v_{22} \text { and } v_{11} v_{22}-v_{12} v_{21}
$$

are solutions of the equations $X_{\lambda}=0$. But

$$
v_{11}+v_{22}=2 I^{\prime}
$$

while 


$$
K=v_{11} v_{22}-v_{12} v_{21},
$$

is obviously a new seminvariant, independent of $I, J, d I / d x, d J / d x$.

If we should now write down the differential equations satisfied by the seminvariants involving $p_{i k}^{(3)}$ and $q_{i k}^{(2)}$ besides the quantities already considered, there would be twenty such equations with only one relation between them, and twenty-eight independent variables. Hence there must be $28-19=9$ such seminvariants. But we know eight of these, viz. :

$$
I, I^{\prime}, I^{\prime \prime} ; J, J^{\prime}, J^{\prime \prime} ; K, K^{\prime} ;
$$

these are independent, for it is easily seen that from the existence of a relation between them would follow the existence of a relation between $I, I^{\prime}, J, J, K$. But these quantities were independent.

We can obtain the ninth semivariant without writing down and integrating the last mentioned system of twenty equations. The process which we shall employ is much more instructive, and is capable of generalization.

We notice first this theorem: the quantities $u_{i k}$ and $v_{i k}$ are cogredient.

This follows from equations (33), together with the corresponding equations for $X_{\lambda}\left(u_{i k}\right)$, as well as from the following formulæ, which express the infinitesimal transformations of $u_{i k}$ and $v_{i k}$. It will be found from (16) and (20), that

$$
\left\{\begin{array}{l}
\frac{\delta u_{11}}{\delta t}=\phi_{21} u_{12}-\phi_{12} u_{21}, \\
\frac{\delta u_{12}}{\delta t}=\left(\phi_{22}-\phi_{11}\right) u_{12}+\phi_{12}\left(u_{11}-u_{22}\right) \\
\frac{\delta u_{21}}{\delta t}=\left(\phi_{11}-\phi_{22}\right) u_{21}+\phi_{21}\left(u_{22}-u_{11}\right), \\
\frac{\delta u_{22}}{\delta t}=-\phi_{21} u_{12}+\phi_{12} u_{21}
\end{array}\right.
$$

and from (16), (32), and (37),

$$
\left\{\begin{array}{l}
\frac{\delta v_{11}}{\delta t}=\phi_{21} v_{12}-\phi_{12} v_{21} \\
\frac{\delta v_{12}}{\delta t}=\left(\phi_{22}-\phi_{11}\right) v_{12}+\phi_{12}\left(v_{11}-v_{22}\right), \\
\frac{\delta v_{21}}{\delta t}=\left(\phi_{11}-\phi_{22}\right) v_{21}+\phi_{21}\left(v_{22}-v_{11}\right), \\
\frac{\delta v_{22}}{\delta t}=-\phi_{21} v_{12}+\phi_{12} v_{21} .
\end{array}\right.
$$


Now certain combinations of the $u_{i k}$ 's and $p_{i k}$ 's, viz. $v_{11}+v_{22}$ and $v_{11} v_{22}-v_{12} v_{21}$, were seminvariants. Since the $v_{i k}$ 's are cogredient with the $u_{i k}$ 's, the same combinations with $v_{i k}$ in place of $u_{i k}$ will also be seminvariants.

Let us, therefore, put

$$
\left\{\begin{array}{l}
w_{11}=2 v_{11}^{\prime}+p_{12} v_{21}-p_{21} v_{12} \\
w_{12}=2 v_{12}^{\prime}+\left(p_{11}-p_{22}\right) v_{12}-p_{12}\left(v_{11}-v_{22}\right) \\
w_{21}=2 v_{21}^{\prime}-\left(p_{11}-p_{22}\right) v_{21}+p_{21}\left(v_{11}-v_{22}\right) \\
w_{22}=2 v_{22}^{\prime}-p_{12} v_{21}+p_{21} v_{12}
\end{array}\right.
$$

then we know that $w_{11}+w_{22}$ and $w_{11} w_{22}-w_{12} w_{21}$ are seminvariants. But

while

$$
w_{11}+w_{22}=2\left(v_{11}^{\prime}+v_{22}^{\prime}\right)=4 I^{\prime},
$$

$$
L=w_{11} w_{22}-w_{12} w_{21}
$$

is the new seminvariant. That it is independent of the other eight can be easily seen by considering the special case in which $p_{i k}=0$.

We now have all the seminvariants, viz.: $I, J, K, L$, and their derivatives. For, suppose we wish to find the seminvariants involving $p_{i k}^{(4)}$ and $q_{i k}^{(3)}$. They are determined by a system of $24-1=23$ independent equations with 36 independent variables. Therefore, there exist $36-23=13$ such seminvariants. But they are merely the 8 seminvariants (36) and $L, I^{\prime \prime \prime}, J^{\prime \prime \prime}, K^{\prime \prime}, L^{\prime}$.

Thus all seminvariants of the system (1) for $m=n=2$, are functions of the quantities $I, J, K, L$, and of their derivatives.

It is interesting to note what would be the result of continuing our above process for obtaining seminvariants. Suppose we had formed

Then would

$$
t_{11}=2 w_{11}^{\prime}+p_{12} w_{21}-p_{21} w_{12}, \text { etc. }
$$

$$
\left\{\begin{array}{l}
t_{11}-t_{22}=g_{1}\left(u_{11}-u_{22}\right)+g_{2}\left(v_{11}-v_{22}\right)+g_{3}\left(w_{11}-w_{22}\right), \\
t_{12}=g_{1} u_{12}+g_{2} v_{12}+g_{3} w_{12} \\
t_{21}=g_{1} u_{21}+g_{2} v_{21}+g_{3} w_{21}
\end{array}\right.
$$

where $g_{1}, g_{2}, g_{3}$ are seminvariants.

For $g_{1}, g_{2}, g_{3}$ are the quotients of determinants of the third order formed out of the matrix

$$
\left|\begin{array}{rrrr}
t_{11}-t_{22}, & u_{11}-u_{22}, & v_{11}-v_{22}, & w_{11}-w_{22} \\
t_{12}, & u_{12}, & v_{12}, & w_{12} \\
t_{21}, & u_{21}, & v_{21}, & w_{21}
\end{array}\right|
$$


and an account of the cogrediency of these quantities, such quotients are seminvariants. In fact, the determinants themselves are seminvariants.

\section{§4. An application of the theory of seminvariants.}

The theory, developed in the preceding paragraphs, was first suggested by the following considerations. Let

$$
D(y)=\frac{d^{n} y}{d x^{n}}+p_{1} \frac{d^{n-1} y}{d x^{n-1}}+\cdots+p_{n} y=0
$$

be a homogeneous, linear, differential equation, and let $y_{1}, \cdots, y_{n}$ form a fundamental system of (41), so that

$$
D\left(y_{i}\right)=0 \quad(i=1,2, \cdots, n) .
$$

Suppose that the coefficients of (41) are uniform functions of $x$, and let $x=a_{\mu}$ be a singular point of one or all of these coefficients. If the variable $x$ describes a closed circuit around this singular point $a_{\mu}, y_{1}, \ldots, y_{n}$ will, in general, undergo a linear substitution with constant coefficients, changing into

$$
\bar{y}_{k}=\sum_{i=1}^{n} \lambda_{k i}^{(\mu)} y_{i}
$$

$(k=1,2, \cdots, n)$,

where the determinant $\left|\lambda_{k i}^{(\mu)}\right| \neq 0$. Denote this substitution by $A_{\mu}$, so that we may write

Now let us put in $(41 a)$

where again the determinant

$$
y_{k}=A_{\mu} y_{k}
$$

$$
y_{k}=\sum_{i=1}^{n} a_{k i} \eta_{i}=S \eta_{k} \quad(k=1,2, \cdots, n),
$$

$$
\left|a_{k i}\right| \neq 0 \text {, }
$$

and where $a_{k i}$ are uniform functions of $x$. Then $\eta_{1}, \cdots, \eta_{n}$ will verify a system of $n$ linear differential equations, obtained from (41a) by the transformation (43). This system is not a general system but has the special property that corresponding to a circuit of $x$ around $a_{\mu}, \eta_{1}, \cdots, \eta_{n}$ undergo the substitution

$$
S^{-1} A_{\mu} S
$$

which is the transformed of $A_{\mu}$ by the substitution $S$, and has as its coefficients uniform functions of $x$.

Conversely, if a system of $n$ linear differential equations has this property, it is obviously possible to find a substitution

$$
y_{k}=S \eta_{k}
$$

which reduces it to the form $(41 a)$, or what amounts to the same thing, $n$ linear 
combinations of $\eta_{1}, \cdots, \eta_{n}$ can be formed with variable coefficients which form a fundamental system of a single linear differential equation of the $n$th order. Such functions as $\eta_{1}, \cdots, \eta_{n}$ are a very special kind of what $\mathrm{I}$ have called $\Lambda$ functions.*

The question then arises: what are the necessary and sufficient conditions in order that a system of linear differential equations may have the property in question?

In our particular case, $m=n=2$, it must then be possible to transform the given system into one of the form

$$
y_{i}^{\prime \prime}+p y_{i}^{\prime}+q y_{i}=0
$$

by a transformation of the form (43). The seminvariants of the given system must therefore be equal to those of (44). But for (44) we have

$$
p_{12}=p_{21}=q_{12}=q_{21}=0, \quad p_{11}=p_{22}=p, \quad q_{11}=q_{22}=q,
$$

so that

whence

$$
\begin{cases}u_{11}=u_{22}=2 p^{\prime}-4 q+p^{2}, & u_{12}=u_{21}=0 \\ v_{11}=v_{22}=2 u_{11}^{\prime}, & v_{12}=v_{21}=0 \\ w_{11}=w_{22}=2 v_{11}^{\prime}=4 u_{11}^{\prime \prime}, & w_{12}=w_{21}=0\end{cases}
$$

$$
I=2 u_{11}, \quad J=u_{11}^{2}, \quad K=4\left(u_{11}^{\prime}\right)^{2}, \quad L=16\left(u_{11}^{\prime \prime}\right)^{2} .
$$

We have then in this case the relations:

$$
I^{2}-4 J=0, \quad K-\left(\frac{d I}{d x}\right)^{2}=0, \quad L-4\left(\frac{d^{2} I}{d x^{2}}\right)^{2}=0 .
$$

As will be seen from the expression deduced for the invariants in $\S 5$, all of the invariants vanish in this case.

But the vanishing of all of the invariants, or even the fulfillment of the equations (48), while necessary, are not sufficient conditions for this case. The conditions (46) however, from which the others were derived, are both necessary and sufficient. It suffices to write down the system of invariant equations

$$
u_{11}-u_{22}=0, \quad u_{12}=u_{21}=0,
$$

for the other equations (46) follow from these.

That the conditions (49) are sufficient follows from the results of $\S 6$. It will there be shown that every system of linear differential equations of the second order can by a transformation of the form

$$
y_{i}=a_{i 1} \eta_{1}+a_{i 2} \eta_{2}
$$$$
(i=1,2) \text {, }
$$

\footnotetext{
*American Journal of Mathematics, rol. 16, No. 2, 1899.
} 
be reduced to a form called the semi-canonical form. The result of this reduction in general is the system (82), which in our case reduces to

$$
\eta_{i}^{\prime \prime}=\frac{1}{4} u_{11} \eta_{i}
$$$$
(i=1,2),
$$

i. e., $\eta_{1}$ and $\eta_{2}$ satisfy the same linear differential equation of the second order.

Thus we have seen that a system of the form

$$
y_{i}^{\prime \prime}+p_{i 1} y_{1}^{\prime}+p_{i 2} y_{2}^{\prime}+q_{i 1} y_{1}+q_{i 2} y_{2}=0
$$$$
(i=1,2)
$$

can, by a transformation of form (50), be reduced to the form (44), if and only if the conditions (49) are fulfilled. In that case, the integration of the system (52) is equivalent to the integration of a single linear differential equation of the second order. The invariants are all zero, and by a change of both dependent and independent variables this equation may be reduced to the form

$$
\frac{d^{2} u}{d \xi^{2}}=0
$$

§5. Calculation of the invariants for $m=n=2$.

The invariants of our system must obviously be functions of the seminvariants which we have already found. We shall therefore first investigate how these seminvariants are affected by a transformation of the independent variable $x$.

As before, let

$$
y_{i}^{\prime \prime}+p_{i 1} y_{1}^{\prime}+p_{i 2} y_{2}^{\prime}+q_{i 1} y_{1}+q_{i 2} y_{2}=0 \quad(i=1,2)
$$

be the given system. If we introduce the new independent variable

this system becomes

$$
\xi=\xi(x) \text {, }
$$

$$
y_{i}^{\prime \prime}+\pi_{i 1} y_{1}^{\prime}+\pi_{i 2} y_{2}^{\prime}+\kappa_{i 1} y_{1}+\kappa_{i 2} y_{2}=0 \quad(i=1,2),
$$

where

$$
\left\{\begin{array}{c}
\pi_{11}=\frac{p_{11}}{\xi^{\prime}}+\frac{\xi^{\prime \prime}}{\left(\xi^{\prime}\right)^{2}}, \quad \pi_{12}=\frac{p_{12}}{\xi^{\prime}}, \quad \pi_{21}={ }_{21}^{\xi^{\prime}}, \quad \pi_{22}=\frac{p_{22}}{\xi^{\prime}}+\frac{\xi^{\prime \prime}}{\left(\xi^{\prime}\right)^{2}}, \\
\kappa_{i k}=\frac{q_{i k}}{\left(\xi^{\prime}\right)^{2}} .
\end{array}\right.
$$

For an infinitesimal transformation, we put

$$
\xi(x)=x+\phi(x) \delta t, \quad \delta x=\phi(x) \delta t,
$$

where $\delta t$ is an infinitesimal and $\phi(x)$ an arbitrary function. We shall then have

$$
\left\{\begin{array}{c}
\delta p_{11}=\left(-\phi^{\prime} p_{11}+\phi^{\prime \prime}\right) \delta t, \quad \delta p_{12}=-\phi^{\prime} p_{12} \delta t \\
\delta p_{21}=-\phi^{\prime} p_{21} \delta t, \quad \delta p_{22}=\left(-\phi^{\prime} p_{22}+\phi^{\prime \prime}\right) \delta t \\
\delta q_{i k}=-2 \phi^{\prime} q_{i k} \delta t .
\end{array}\right.
$$


If $f$ is any function of $x$, and $f^{\prime}$ its derivative, we have

$$
\delta f^{\prime}=\delta \frac{d f}{d x}=\frac{\delta(d f)}{d x}-\frac{d f}{d x^{2}} \delta(d x)=\frac{d}{d x}(\delta f)-f^{\prime} \frac{d}{d x}(\delta x)
$$

or

$$
\delta f^{\prime}=\frac{d}{d x}(\delta f)-f^{\prime} \phi^{\prime}(x) \delta t .
$$

Thus we find from (58)

$$
\left\{\begin{array}{l}
\delta p_{11}^{\prime}=\left(\phi^{(3)}-\phi^{\prime \prime} p_{11}-2 \phi^{\prime} p_{11}^{\prime}\right) \delta t \\
\delta p_{12}^{\prime}=\left(-\phi^{\prime \prime} p_{12}-2 \phi^{\prime} p_{12}^{\prime}\right) \delta t \\
\delta p_{21}^{\prime}=\left(-\phi^{\prime \prime} p_{21}-2 \phi^{\prime} p_{21}^{\prime}\right) \delta t \\
\delta p_{22}^{\prime}=\left(\phi^{(3)}-\phi^{\prime \prime} p_{22}-2 \phi^{\prime} p_{22}^{\prime}\right) \delta t
\end{array}\right.
$$

Therefore, making use of $(20),(58)$, and $(60)$, we find

$$
\begin{cases}\delta u_{11}=\left(2 \phi^{(3)}-2 \phi^{\prime} u_{11}\right) \delta t, & \delta u_{12}=-2 \phi^{\prime} u_{12} \delta t \\ \delta u_{21}=-2 \phi^{\prime} u_{21} \delta t & , \quad \delta u_{22}=\left(2 \phi^{(3)}-2 \phi^{\prime} u_{22}\right) \delta t .\end{cases}
$$

Consequently we shall have, remembering the definition of $I$ and $J$,

$$
\left\{\begin{array}{l}
\delta I=\left(4 \phi^{(3)}-2 \phi^{\prime} I\right) \delta t, \\
\delta J=\left(2 \phi^{(3)} I-4 \phi^{\prime} J\right) \delta t .
\end{array}\right.
$$

In the same way we find

whence

$$
\left\{\begin{array}{l}
\delta v_{11}=\left(4 \phi^{(4)}-4 \phi^{\prime \prime} u_{11}-3 \phi^{\prime} v_{11}\right) \delta t \\
\delta v_{12}=\left(-4 \phi^{\prime \prime} u_{12}-3 \phi^{\prime} v_{12}\right) \delta t \\
\delta v_{21}=\left(-4 \phi^{\prime \prime} u_{21}-3 \phi^{\prime} v_{21}\right) \delta t \\
\delta v_{22}=\left(4 \phi^{(t)}-4 \phi^{\prime \prime} u_{22}-3 \phi^{\prime} v_{22}\right) \delta t
\end{array}\right.
$$

$$
\delta K=\left(8 \phi^{(4)} I^{\prime}-8 \phi^{\prime \prime} J^{\prime}-6 \phi^{\prime} K\right) \delta t,
$$

after a slight transformation, involving equation (67) for $J^{\prime}$.

We find further

$$
\left\{\begin{array}{l}
\delta w_{11}=\left(8 \phi^{(5)}-8 \phi^{(3)} u_{11}-10 \phi^{\prime \prime} v_{11}-4 \phi^{\prime} w_{11}\right) \delta t \\
\delta w_{12}=\left(-8 \phi^{(3)} u_{12}-10 \phi^{\prime \prime} v_{12}-4 \phi^{\prime} w_{12}\right) \delta t \\
\delta w_{21}=\left(-8 \phi^{(3)} u_{21}-10 \phi^{\prime \prime} v_{21}-4 \phi^{\prime} w_{21}\right) \delta t \\
\delta w_{22}=\left(8 \phi^{(5)}-8 \phi^{(3)} u_{22}-10 \phi^{\prime \prime} v_{22}-4 \phi^{\prime} w_{22}\right) \delta t
\end{array}\right.
$$

whence follows 


$$
\delta L=\left[32 \phi^{(5)} I^{\prime \prime}-16 \phi^{(3)}\left(2 J^{\prime \prime}-K\right)-20 \phi^{\prime \prime} K^{\prime}-8 \phi^{\prime} L\right] \delta t,
$$

if it be noted that

$$
\left\{\begin{array}{l}
u_{11} v_{22}+u_{22} v_{11}-u_{12} v_{21}-u_{21} v_{12}=2 J^{\prime} \\
v_{11} w_{22}+v_{22} w_{11}-v_{12} w_{21}-v_{21} w_{12}=2 K^{\prime} \\
w_{11} u_{22}+w_{22} u_{11}-w_{12} u_{21}-w_{21} u_{12}=2\left(2 J^{\prime \prime}-K\right) .
\end{array}\right.
$$

Let us now, by an obvious extension of the theory of invariants of a single linear differential equation, assign to $p_{i k}$ the weight -1 , to $q_{i k}$ the weight -2 , while to $p_{i k}^{(\lambda)}$ and $q_{i k}^{(\mu)}$ are assigned the weights $-1-\lambda,-2-\mu$ respectively. Then it is easy to see that the following statements are true.

1. Every absolute invariant is isobaric in the coefficients and of weight zero.

2. If an absolute invariant is a rational function of the coefficients and of their derivatives, it is the quotient of two relative invariants of the same weight.

3. A relative invariant is isobaric in the coefficients, and if the common weight of all of its terms is $-\nu$, it satisfies the equation

$$
\xi^{\prime}(x)^{\nu} \theta_{\nu}(\xi)=\theta_{\nu}(x),
$$

or, for infinitesimal transformations,

$$
\delta \theta_{\nu}=-\nu \phi^{\prime}(x) \theta_{\nu} \delta t
$$

We shall speak of such an invariant as being of weight $\nu$, rather than $-\nu$. The negative weights have been introduced, following the example of ForsYTH, principally because they have a decided advantage when we consider covariants.

The proof of the above three statements is essentially the same as in the case of a single linear differential equation, and need not, therefore, be given.

It is now a simple matter to find the rational invariants of any assigned weight, or else to establish their non-existence.

First, it is clear that no such invariants of weights 1, 2, 3 exist. An invariant of weight 4 must verify the equation

The invariant is

$$
\begin{gathered}
\delta \theta_{4}=-4 \phi^{\prime}(x) \theta_{4} \delta t . \\
\theta_{4}=I^{2}-4 J .
\end{gathered}
$$

An invariant of weight 5 must be of the form

$$
a I I^{\prime}+b I^{(3)}+c J^{\prime}
$$

We find that no such invariant exists, or that it vanishes identically. 
An invariant of weight 6 must verify the equation

$$
\frac{\delta \theta_{6}}{\delta t}=-6 \phi^{\prime} \theta_{6} \text {. }
$$

The most general expression of weight 6 is

We find

$$
\theta_{6}=a I^{3}+b I J+c K+d I^{(4)}+e J^{\prime \prime}+f I I^{\prime \prime}+g I^{\prime 2} \text {. }
$$

$$
\begin{aligned}
\frac{\delta \theta_{6}}{\delta t}=3 a I^{2} & \left(4 \phi^{(3)}-2 \phi^{\prime} I\right)+b I\left(2 \phi^{(3)} I-4 \phi^{\prime} J\right)+b J\left(4 \phi^{(3)}-2 \phi^{\prime} I\right) \\
& +c\left(8 \phi^{(4)} I^{\prime}-8 \phi^{\prime \prime} J-6 \phi^{\prime} K\right) \\
& +d\left(4 \phi^{(7)}-2 \phi^{(5)} I-9 \phi^{(4)} I^{\prime}-12 \phi^{(3)} I^{\prime \prime}-10 \phi^{\prime \prime} I^{(3)}-6 \phi^{\prime} I^{(4)}\right) \\
& +e\left(2 \phi^{(5)} I+4 \phi^{(4)} I^{\prime}+2 \phi^{(3)} I^{\prime \prime}-4 \phi^{(3)} J-9 \phi^{\prime \prime} J^{\prime}-6 \phi^{\prime} J^{\prime \prime}\right) \\
& +f I\left(4 \phi^{(5)}-2 \phi^{(3)} I-5 \phi^{\prime \prime} I^{\prime}-4 \phi^{\prime} I^{\prime \prime}\right)+f I^{\prime \prime}\left(4 \phi^{(3)}-2 \phi^{\prime} I\right) \\
& +2 g I^{\prime}\left(4 \phi^{(4)}-2 \phi^{\prime \prime} I-3 \phi^{\prime} I^{\prime}\right),
\end{aligned}
$$

and this must be equal to $-6 \phi^{\prime} \theta_{6}$ for all values of $\phi, \phi^{\prime}, \ldots, I, I^{\prime}, \ldots, J, J$, $\ldots, K$. We find therefore the equations :

whence

$$
\left\{\begin{array}{l}
d=0, \quad e+2 f=0, \quad 2 c+e+2 g=0, \quad 6 a+b-f=0, \\
b-e=0, \quad 8 c+9 e=0, \quad 5 f+4 g=0,
\end{array}\right.
$$

$$
a=-\frac{1}{4} e, \quad b=e, \quad c=-\frac{9}{8} e, \quad d=0, \quad f=-\frac{1}{2} e, \quad g=\frac{5}{8} e .
$$

Putting $e=-8$, we find

$$
\theta_{6}=2 I\left(I^{2}-4 J\right)+5\left(K-I^{2}\right)+4\left(K-2 J^{\prime \prime}+I I^{\prime \prime}\right) .
$$

There is no invariant of weight 7 , and there are two independent invariants of weight 8 , one of which is $\theta_{4}^{2}$, while the other is

$$
\begin{aligned}
\theta_{8}= & 143\left(L-4 I^{\prime 2}\right)-54\left(I^{2}+4 J\right) \theta_{4}-20 I^{\prime \prime} \theta_{4}+25 I^{\prime} \theta_{4}^{\prime}-206 I \theta_{4}^{\prime \prime} \\
& -20 \theta_{4}^{(4)}-902 I\left(K-I^{\prime 2}\right)-220\left(K^{\prime \prime}-2 I^{\prime} I^{(3)}-2 I^{\prime \prime 2}\right) .
\end{aligned}
$$

We can easily find an invariant of weight 10 , without going through this general process. We have

$$
\begin{aligned}
& \frac{\delta\left(K-I^{\prime 2}\right)}{\delta t}=4 \phi^{\prime \prime}\left(I I^{\prime}-2 J^{\prime}\right)-6 \phi^{\prime}\left(K-{I^{\prime}}^{2}\right), \\
& \frac{\delta\left(I I^{\prime}-2 J^{\prime}\right)}{\delta t}=-2 \phi^{\prime \prime}\left(I^{2}-4 J\right)-5 \phi^{\prime}\left(I I^{\prime}-2 J^{\prime}\right),
\end{aligned}
$$


whence eliminating $\phi^{\prime \prime}$, we find

where

$$
\frac{\delta \theta_{10}}{\delta t}=-10 \phi^{\prime} \theta_{10}
$$

$$
\theta_{10}=\left(I^{2}-4 J\right)\left(K-I^{\prime 2}\right)+\left(I I^{\prime}-2 J^{\prime}\right)^{2} .
$$

From any two invariants $\theta_{\lambda}$ and $\theta_{\mu}$, of weights $\lambda$ and $\mu$ respectively, we can always deduce a third. For we have

$$
\frac{\theta_{\lambda}^{\mu}(\xi)}{\theta_{\mu}^{\lambda}(\xi)}=\frac{\theta_{\lambda}^{\mu}(x)}{\theta_{\mu}^{\lambda}(x)}
$$

an absolute invariant. Hence, by logarithmic differentiation,

Therefore

$$
\left\{\mu \frac{\theta_{\lambda}^{\prime}(\xi)}{\theta_{\lambda}(\xi)}-\lambda \frac{\theta_{\mu}^{\prime}(\xi)}{\theta_{\mu}(\xi)}\right\} \xi^{\prime}=\mu \frac{\theta_{\lambda}^{\prime}(x)}{\theta_{\lambda}(x)}-\lambda \frac{\theta_{\mu}^{\prime}(x)}{\theta_{\mu}(x)}
$$

$$
\theta_{\lambda+\mu+1}=\mu \theta_{\mu} \theta_{\lambda}^{\prime}-\lambda \theta_{\lambda} \theta_{\mu}^{\prime}
$$

is a new invariant of weight $\lambda+\mu+1$. It is called by ForsYTH the Jacobian of $\theta_{\lambda}$ and $\theta_{\mu}$, in his theory of invariants of a single equation.

We thus obtain

$$
\left\{\begin{array}{l}
\theta_{11}=3 \theta_{6} \theta_{4}^{\prime}-2 \theta_{4} \theta_{6}^{\prime}, \\
\theta_{13}=2 \theta_{8} \theta_{4}^{\prime}-\theta_{4} \theta_{8}^{\prime}, \\
\theta_{15}=5 \theta_{10} \theta_{4}^{\prime}-2 \theta_{4} \theta_{10}^{\prime}, \\
\theta_{15}=4 \theta_{8} \theta_{6}^{\prime}-3 \theta_{6} \theta_{8}^{\prime}, \\
\theta_{17}=5 \theta_{10} \theta_{6}^{\prime}-3 \theta_{6} \theta_{10}^{\prime}, \\
\theta_{19}=5 \theta_{10} \theta_{8}^{\prime}-4 \theta_{8} \theta_{10}^{\prime},
\end{array}\right.
$$

from which still others can be derived. Of all of the invariants found so far

$$
\theta_{4}, \theta_{6}, \theta_{10}, \theta_{15}
$$

are the only ones which involve no higher derivatives of $p_{i k}$ than the third, and no higher derivatives of $q_{i k}$ than the second, or what amounts to the same thing, these are the only invariants found so far which depend only on the seminvariants

$$
I, I^{\prime}, I^{\prime \prime} ; J, J^{\prime}, J^{\prime \prime} ; K, K^{\prime} ; L \text {. }
$$

Moreover the invariants (74) are not all independent. For instance we have the syzygy 


$$
2 \theta_{4} \theta_{17}+5 \theta_{10} \theta_{11}-6 \theta_{6} \theta_{15}=0 .
$$

In order to obtain all of the invariants depending only upon the seminvariants (75), we write down the partial differential equations which such invariants must verify.

From the expressions for $\delta I, \delta I^{\prime}, \delta I^{\prime \prime} . \delta J$, etc., $\delta L$, we find that these partial differential equations are five in number, viz. :

$$
\left\{\begin{array}{c}
Y_{1} f=2 I \frac{\partial f}{\partial I}+3 I^{\prime} \frac{\partial f}{\partial I^{\prime}}+4 I^{\prime \prime} \frac{\partial f}{\partial I^{\prime \prime}}+4 J^{\prime} \frac{\partial f}{\partial J}+5 J^{\prime} \frac{\partial f}{\partial J^{\prime}}+6 J^{\prime \prime} \frac{\partial f}{\partial J^{\prime \prime}} \\
+6 K \frac{\partial f}{\partial K}+7 K^{\prime} \frac{\partial f}{\partial K^{\prime}}+8 L \frac{\partial f}{\partial L}=0 \\
Y_{2} f=-2 I \frac{\partial f}{\partial I^{\prime}}-5 I^{\prime} \frac{\partial f}{\partial I^{\prime \prime}}-4 J \frac{\partial f}{\partial J^{\prime}}-9 J^{\prime} \frac{\partial f}{\partial J^{\prime \prime}}-8 J^{\prime} \frac{\partial f}{\partial K} \\
-\left(8 J^{\prime \prime}+6 K\right) \frac{\partial f}{\partial K^{\prime}}-20 K^{\prime} \frac{\partial f}{\partial L}=0 \\
\quad-8 J^{\prime} \frac{\partial f}{\partial K^{\prime}}-16\left(2 J^{\prime \prime}-K\right) \frac{\partial f}{\partial L}=0 \\
Y_{3} f=4 \frac{\partial f}{\partial I}-2 I \frac{\partial f}{\partial I^{\prime \prime}}+2 I \frac{\partial f}{\partial J}+2 I^{\prime} \frac{\partial f}{\partial J^{\prime}}+2\left(I^{\prime \prime}-2 J\right) \frac{\partial f}{\partial J^{\prime \prime}} \\
Y_{4} f=4 \frac{\partial f}{\partial I^{\prime}}+2 I \frac{\partial f}{\partial J^{\prime}}+4 I^{\prime} \frac{\partial f}{\partial J^{\prime \prime}}+8 I^{\prime} \frac{\partial f}{\partial K}+8 I^{\prime \prime} \frac{\partial f}{\partial K^{\prime}}=0 \\
Y_{5} f=4 \frac{\partial f}{\partial I^{\prime \prime}}+2 I \frac{\partial f}{\partial J^{\prime \prime}}+8 I^{\prime} \frac{\partial f}{\partial K^{\prime}}+32 I^{\prime \prime} \frac{\partial f}{\partial L}=0,
\end{array}\right.
$$

where $Y_{i} f=0$ is obtained by equating to zero the coefficient of $\phi^{(i)}$ in $\delta f$.

They are independent and, therefore, have $9-5=4$ independent solutions, i. e., there are four independent absolute, or five independent relative invariants. We have already found four of these, viz. : $\theta_{4}, \theta_{6}, \theta_{10}, \theta_{15}$. The fifth invariant, found by integrating (77), is

$$
\theta_{22}=\theta_{10}\left[\left(I^{2}-4 J\right)\left(L-4 I^{\prime \prime 2}\right)+4\left(I I^{\prime \prime}-2 J^{\prime \prime}+K\right)^{2}\right]-\theta_{10}^{\prime 2} .
$$

But this can be shown to be divisible by $\theta_{4}$, so that we may complete our system of invariants by taking $\theta_{22} / \theta_{4}$, which is

$$
\begin{aligned}
\theta_{18}=\theta_{10}\left(L-4 I^{\prime \prime 2}\right)+4\left(K-I^{\prime 2}\right) & \left(I I^{\prime \prime}-2 J^{\prime \prime}+K\right)^{2}-\theta_{4}\left(K^{\prime}-2 I^{\prime} I^{\prime \prime}\right)^{2} \\
& -2 \theta_{4}^{\prime}\left(K^{\prime}-2 I^{\prime} I^{\prime \prime}\right)\left(I I^{\prime \prime}-2 J^{\prime \prime}+K\right) .
\end{aligned}
$$


§6. Canonical forms of a system of two linear differential equations of the second order.

Our system of equations (54) can always be transformed into another which contains no first derivatives, by a transformation of the form

$$
y_{i}=a_{i 1} \eta_{1}+a_{i 2} \eta_{2} \quad(i=1,2),
$$

where $a_{i k}$ are functions of $x$. For, on making this transformation, we find

$$
\left\{\begin{aligned}
a_{i 1} \eta_{1}^{\prime \prime}+ & a_{i 2} \eta_{2}^{\prime \prime}+\left(2 a_{i 1}^{\prime}+p_{i 1} a_{11}+p_{i 2} a_{21}\right) \eta_{1}^{\prime}+\left(2 a_{i 2}^{\prime}+p_{i 1} a_{12}+p_{i 2} a_{22}\right) \eta_{2}^{\prime} \\
& +\left(a_{i 1}^{\prime \prime}+p_{i 1} a_{11}^{\prime}+p_{i 2} a_{21}^{\prime}+q_{i 1} a_{11}+q_{i 2} a_{21}\right) \eta_{1} \\
& +\left(a_{i 2}^{\prime \prime}+p_{i 1} a_{12}^{\prime}+p_{i 2} a_{22}^{\prime}+q_{i 1} a_{12}+q_{i 2} a_{22}\right) \eta_{2}=0 \quad(i=1,2),
\end{aligned}\right.
$$

so that, if we take $a_{i k}$ subject to the conditions

$$
\begin{cases}a_{11}^{\prime}=-\frac{1}{2}\left(p_{11} a_{11}+p_{12} a_{21}\right), & a_{12}^{\prime}=-\frac{1}{2}\left(p_{11} a_{12}+p_{12} a_{22}\right), \\ a_{21}^{\prime}=-\frac{1}{2}\left(p_{21} a_{11}+p_{22} a_{21}\right), & a_{22}^{\prime}=-\frac{1}{2}\left(p_{21} a_{12}+p_{22} a_{22}\right),\end{cases}
$$

equations (79) will contain no terms in $\eta_{1}^{\prime}$ and $\eta_{2}^{\prime}$. The functions $\left(a_{11}, a_{21}\right)$ and $\left(a_{12}, a_{22}\right)$ must therefore be taken as solutions of the same system of linear differential equations, viz.,

$$
\left\{\begin{array}{l}
a^{\prime}=-\frac{1}{2}\left(p_{11} a+p_{12} \beta\right), \\
\beta^{\prime}=-\frac{1}{2}\left(p_{21} a+p_{22} \beta\right) .
\end{array}\right.
$$

Moreover $\left(a_{11}, a_{21}\right)$ and $\left(a_{12}, a_{22}\right)$ must be independent solutions of (81), for otherwise the determinant

$$
a_{11} a_{22}-a_{12} a_{21}
$$

would vanish. The functions $a_{i k}$ can therefore be determined so as to effect the desired result, by integrating (81).

If one makes use of (80) and the equations obtained from (80) by differentiation the transformed system (79) becomes

$$
\left\{\begin{array}{l}
a_{11} \eta_{1}^{\prime \prime}+a_{12} \eta_{2}^{\prime \prime}=\frac{1}{4}\left(a_{11} u_{11}+a_{21} u_{12}\right) \eta_{1}+\frac{1}{4}\left(a_{12} u_{11}+a_{22} u_{12}\right) \eta_{2}, \\
a_{21} \eta_{1}^{\prime \prime}+a_{22} \eta_{2}^{\prime \prime}=\frac{1}{4}\left(a_{11} u_{21}+a_{21} u_{22}\right) \eta_{1}+\frac{1}{4}\left(a_{12} u_{21}+a_{22} u_{22}\right) \eta_{2}
\end{array}\right.
$$

where the quantities $u_{i k}$ have been previously defined.

Thus every binary system of homogeneous linear differential equations of the second order can be converted into another, involving no first derivatives, i. e., into one for which $p_{i k}=0$. We will say that this transformed system has the semi-canonical form.

Suppose we have reduced a system to its semi-canonical form. Let us find the most general sub-group $G^{\prime}$ of our general infinite continuous group $G$, which leaves the semi-canonical form of the system unaltered. 
In the semi-canonical form $p_{i k}=0$. Let us make the most general infinitesimal transformation of the general group $G$. The coefficients of the first derivatives in the transformed system will be, according to (16) and (58),

$$
\begin{cases}\delta p_{11}=\left(2 \phi_{11}^{\prime}-\phi^{\prime \prime}\right) \delta t, & \delta p_{12}=2 \phi_{12}^{\prime} \delta t, \\ \delta p_{21}=2 \phi_{21}^{\prime} \delta t, & \delta p_{22}=\left(2 \phi_{22}^{\prime}-\phi^{\prime \prime}\right) \delta t,\end{cases}
$$

for the infinitesimal transformations of the coefficients due to infinitesimal transformations of both dependent and independent variables are equal to those due to the transformations of the dependent variables alone plus those due to the transformation of the independent variable alone.*

But for all of the transformations of our sub-group $G^{\prime}$, these quantities must vanish. Therefore must

$$
\phi_{12}^{\prime}=\phi_{21}^{\prime}=0,2 \phi_{11}^{\prime}-\phi^{\prime \prime}=0,2 \phi_{22}^{\prime}-\phi^{\prime \prime}=0,
$$

or, if we denote by $c_{i k}$ arbitrary constants,

$$
\begin{cases}\phi_{11}=c_{11}+\frac{1}{2} \phi^{\prime}, & \phi_{12}=c_{12}, \\ \phi_{21}=c_{21}, & \phi_{22}=c_{22}+\frac{1}{2} \phi^{\prime},\end{cases}
$$

so that the sub-group $G^{\prime}$ depends upon 4 arbitrary parameters $c_{i k}$, and an arbitrary function $\phi^{\prime}(x)$.

We can determine a sub-group $G^{\prime \prime}$ of the $\cdot$ subgroup $G^{\prime}$, namely, that which leaves $q_{11}+q_{22}$ invariant.

We have, in the same way as above, according to (16) and (58),

$$
\delta\left(q_{11}+q_{22}\right)=\left[2 \phi^{\prime}\left(q_{11}+q_{22}\right)+\phi_{11}^{(2)}+\phi_{22}^{(2)}\right] \delta t,
$$

so that for this sub-group we have the further relation

$$
\phi_{11}^{(2)}+\phi_{22}^{(2)}=-2 \phi^{\prime}\left(q_{11}+q_{22}\right),
$$

or, using (83),

$$
\phi_{11}^{(2)}+\phi_{22}^{(2)}+2\left[c_{11}+c_{22}-\left(\phi_{11}+\phi_{22}\right)\right]\left(q_{11}+q_{22}\right)=0,
$$

a linear differential equation of the second order for $\phi_{11}+\phi_{22}$. Its integration introduces two arbitrary constants ; then $\phi^{\prime}$ is found from (84), and the quadrature

$$
\phi=\int \phi^{\prime} d x
$$

gives rise to a third constant. Thus $G^{\prime \prime}$ is a seven-parameter group. We can also represent $G^{\prime \prime}$ by $(83)$, together with

$$
-\phi^{(3)}=2 \phi^{\prime}\left(q_{11}+q_{22}\right),
$$

* We have changed the sign of $\phi(x)$, so as to make the infinitesimal transformation of the independent variable harmonize with those of the dependent variables. 
an equation, whose integration gives rise to three constants. These together with the four constants $c_{i / c}$ are the parameters of the group.

The semi-canonical form of the system can be further simplified by the reduction to what we shall call the canonical form.

Suppose we have reduced our system by a first transformation to the semicanonical form

$$
y_{i}^{\prime \prime}+q_{i 1} y_{1}+q_{i 2} y_{2}=0 \quad(i=1,2) .
$$

If now we make the transformation

we find

$$
\xi=\xi(x), \quad y_{i}=\beta_{i 1} \eta_{1}+\beta_{i 2} \eta_{2} \quad(i=1,2),
$$

$$
\begin{aligned}
& \beta_{i 1} \xi^{\prime 2} \frac{d^{2} \eta_{1}}{d \xi^{2}}+\beta_{i 2} \xi^{\prime 2} \frac{d^{2} \eta_{2}}{d \xi^{2}}+\left(\beta_{i 1} \xi^{\prime \prime}+2 \beta_{i 1}^{\prime} \xi^{\prime}\right) \frac{d \eta_{1}}{d \xi}+\left(\beta_{i 2} \xi^{\prime \prime}+2 \beta_{i 2}^{\prime} \xi^{\prime}\right) \frac{d \eta_{2}}{d \xi} \\
& +\left(\beta_{i 1}^{\prime \prime}+q_{i 1} \beta_{11}+q_{i 2} \beta_{21}\right) \eta_{1}+\left(\beta_{i 2}^{\prime \prime}+q_{i 1} \beta_{12}+q_{i 2} \beta_{22}\right) \dot{\eta}_{2}=0 \quad(i=1,2)
\end{aligned}
$$

which is again in the semi-canonical form if

or

$$
\beta_{i k} \xi^{\prime \prime}+2 \beta_{i k}^{\prime} \xi^{\prime}=0
$$

$$
\beta_{i k}=\frac{e_{i k}}{\sqrt{\xi^{\prime}}},
$$

where $e_{i k}$ are arbitrary constants, whose determinant does not vanish. Put

or

$$
e_{11}=e_{22}=1, \quad e_{12}=e_{21}=0,
$$

$$
\beta_{11}=\beta_{22}=\beta=\frac{1}{\sqrt{\xi^{\prime}}}, \quad \beta_{12}=\beta_{21}=0 \text {. }
$$

Then (88) becomes

$$
\left\{\begin{array}{l}
\beta \xi^{\prime 2} \frac{d^{2} \eta_{1}}{d \xi^{2}}+\left(\beta^{\prime \prime}+q_{11} \beta\right) \eta_{1}+q_{12} \beta \eta_{2}=0 \\
\beta \xi^{\prime 2} \frac{d^{2} \eta_{2}}{d \xi^{2}}+q_{21} \beta \eta_{1}+\left(\beta^{\prime \prime}+q_{22} \beta\right) \eta_{2}=0
\end{array}\right.
$$

or

$$
\frac{d^{2} \eta_{i}}{d \xi^{2}}+\rho_{i 1} \eta_{1}+\rho_{i 2} \eta_{2}=0
$$

Now $\beta$ can be determined that in $(91), \rho_{11}+\rho_{22}=0$; for this purpose it is only necessary to take for $\beta$ a solution of the linear differential equation

$$
2 \beta^{\prime \prime}+\left(q_{11}+q_{22}\right) \beta=0 .
$$

We have proved the following theorem : 
Every system of linear, homogeneous differential equations of the second order can be converted into a system having the canonical form

where

$$
\frac{d^{2} \eta_{i}}{d \xi^{2}}+\rho_{i 1} \eta_{1}+\rho_{i 2} \eta_{2} \quad \quad(i=1,2)
$$

$$
\rho_{11}+\rho_{22}=0 \text {. }
$$

In order to effect this reduction, it is necessary to integrate a system of homogeneous linear differential equaticns of the first order (81), a single homogeneous linear differential equation of the second order (92), and finally to effect the quadrature

$$
\xi(x)=\int \frac{d x}{\beta^{2}}
$$

This canonical form of the system corresponds to the LAGUERRE-ForSYTH form of a single linear differential equation.

The sub-group of $G$ which leaves this canonical form unaltered is especially simple. It is the group $G^{\prime \prime}$ for the particular case that $q_{11}+q_{22}=0$, whence, according to (86),

and from (83),

$$
\phi^{(3)}=0, \quad \text { or } \quad \phi=\lambda+\mu x+\nu x^{2},
$$

$$
\begin{cases}\phi_{11}=c_{11}+\frac{1}{2} \mu+\nu x, & \phi_{12}=c_{12}, \\ \phi_{21}=c_{21}, & \phi_{22}=c_{22}+\frac{1}{2} \mu+\nu x .\end{cases}
$$

The finite transformations of this group are

$$
\xi=\frac{a x+\beta}{\gamma x+\delta}, \quad \eta_{i}=\frac{C_{i 1} y_{1}+C_{i 2} y_{2}}{\gamma x+\delta} \quad(i=1,2) .
$$

The functions called, by Forsyth, quadriderivatives are invariants for this sub-group. If $\theta_{\sigma}$ is an invariant of weight $\sigma$, then

$$
\theta_{\sigma, 1}=2 \sigma \theta_{\sigma} \theta_{\sigma}^{\prime \prime}-(2 \sigma+1) \theta_{\sigma}^{\prime 2}
$$

is what ForsYTH calls the quadriderivative of $\theta_{\sigma}$.

We find that for the general infinitesimal transformations of the general group $G$,

$$
\delta \theta_{\sigma, 1}=-2 \sigma^{2} \theta_{\sigma}^{2} \phi^{(3)} \delta t-2(\sigma+1) \phi^{\prime} \theta_{\sigma, 1} \delta t .
$$

For our sub-group $\phi^{(3)}=0$, so that $\theta_{\sigma, 1}$ is indeed an invariant of the subgroup. Its weight is $2 \sigma+2$. 


\section{§ 7. Covariants.}

We shall not treat of covariants in an exhaustive manner in this paper. It will be sufficient to note a few simple theorems.

First we may observe that no absolute covariant exists which depends only upon $y_{1}, y_{2}, d y_{1} / d x, d y_{2} / d x$, etc., $i$. e., which does not contain $x$ explicitly, and makes no use of the fact that $y_{1}$ and $y_{2}$ are solutions of (54).

For we have, taking infinitesimal transformations of the dependent variables only,

$$
\left\{\begin{aligned}
\delta y_{i} & =\left(\phi_{i 1} y_{1}+\phi_{i 2} y_{2}\right) \delta t, \\
\delta y_{i}^{\prime} & =\left(\phi_{i 1} y_{1}^{\prime}+\phi_{i 2} y_{2}^{\prime}+\phi_{i 1}^{\prime} y_{1}+\phi_{i 2}^{\prime} y_{2}\right) \delta t, \\
\cdot & \left.\cdot \cdot \cdot \cdot \cdot \cdot \cdot \cdot \cdot \cdot \cdot \cdot \cdot \cdot \cdot \cdot \phi_{i 2}^{(m-1)} y_{2}\right) \delta t .
\end{aligned}\right.
$$

But this group in $2 m$ variables is always transitive. For if we take the special case obtained by putting $\phi_{i k}, \phi_{i k}^{\prime}, \phi_{i k}^{\prime \prime}$, etc., equal to arbitrary constants, the group becomes a finite $4 m$ parameter group in $2 m$ variables which is a particular case of a general group of linear substitutions with $m n^{2}$ parameters and $m n$ variables. This general group has been studied by the author and was found to be transitive.* Therefore the more general infinite group is also transitive, i. e., it has no invariants, and thus the theorem is proved.

It is even impossible to construct functions $z_{1}$ and $z_{2}$ of $y_{1}, y_{2}$ and of their successive derivatives, not involving $x$ explicitly, which shall be cogredient with $y_{1}$ and $y_{2}$.

For, if it were not so, from $z_{1}$ and $z_{2}$ could then be constructed a third cogredient set, say $\eta_{1}$ and $\eta_{2}$, and the quotient

$$
\frac{y_{1} z_{2}-y_{2} z_{1}}{y_{1} \eta_{2}-y_{2} \eta_{1}}
$$

would be an absolute covariant of the form whose non-existence we have just shown.

An infinite number of covariants, containing $x$ explicitly, can be constructed as follows. Put

$$
\left\{\begin{array}{c}
\bar{y}_{i}=y_{i}^{\prime \prime}+p_{i 1} y_{1}^{\prime}+p_{i 2} y_{2}^{\prime}+q_{i 1} y_{1}+q_{i 2} y_{2}, \\
\bar{y}_{i}=\bar{y}_{i}^{\prime \prime}+p_{i 1} \bar{y}_{1}^{\prime}+p_{i 2} \bar{y}_{2}^{\prime}+q_{i 1} \bar{y}_{1}+q_{i 2} \bar{y}_{2}, \\
\cdot \cdot \cdot \cdot
\end{array} .\right.
$$

Then $\bar{y}_{i}, \overline{\bar{y}}_{i}$, etc., are cogredient with $y_{i}$. Therefore the determinants

$$
y_{1} \bar{y}_{2}-y_{2} \bar{y}_{1}, \quad y_{1} \bar{y}_{2}-y_{2} \overline{\bar{y}}_{1} \text {, etc., }
$$

\footnotetext{
* Proceedings of the California Academy of Sciences, vol. 1, no..6.
} 
are covariants. All of these vanish in consequence of the given system of differential equations.

Their covariant property is expressed by an equation of the form

where

$$
C\left(\eta_{1}, \eta_{2}, \xi\right)=\frac{a_{11} a_{22}-a_{12} a_{21}}{\xi^{\prime}(x)^{2}} C\left(y_{1}, y_{2}, x\right),
$$

$$
\eta_{i}=a_{i 1} y_{1}+a_{i 2} y_{2}, \quad \xi=\xi(x) \quad(i=1,2),
$$

is the transformation of the variables.

Suppose we have reduced our given system to its canonical form. Then

$$
y_{1} y_{2}^{\prime}-y_{2} y_{1}^{\prime}
$$

is a covariant of the sub-group (93) which leaves this canonical form unchanged. To find all of the covariants of the system we could proceed as in the case of the invariants by setting up the system of partial differential equations which they satisfy. We might also construct functions cogredient with $y_{1}$ and $y_{2}$. The determinant of two such systems would be a covariant.

University of California, Berkeley,

October 6, 1900. 